\title{
Bone Ninja Mobile App for Reverse Planning Method in Internal Limb Deformity and Lengthening Surgery
}

\author{
Alec Lik-Hang Hung ${ }^{1}$, Philip K McClure ${ }^{2}$, Jeanne M Franzone ${ }^{3}$, Ahmed I Hammouda ${ }^{4}$, Shawn C Standard ${ }^{5}$, Wai-Wang Chau ${ }^{6}$, \\ John E Herzenberg ${ }^{7}$
}

\begin{abstract}
Aim: To report whether Bone Ninja (BN) is a reliable tool to teach the reverse planning method (RPM) for implantable intramedullary (IM) limblengthening devices and for deformity correction surgery.

Background: Motorised fully implantable implantable intramedullary (IM) lengthening devices have been gaining popularity all over the world for limb-lengthening procedures. Multiple advantages have been demonstrated over external fixator-controlled lengthening. Mechanical axis deviation may result if careful preoperative planning and surgical intervention are not completed for femur cases. The RPM proposed by Baumgart has been shown to be an accurate means of arriving at the desired end point. The RPM addresses the ideal correction position accounting for length, angulation, and translation created during lengthening along the nail axis. The original description calls for the use of life-size paper tracings of the bone and large light boxes to allow planning. We propose an alternative method using a digital tool that is readily available. The BN mobile app was developed for patient/physician education and is available for the Apple iPad platform. Bone Ninja has been shown to have similar accuracy for measurements of the limb length and deformity angles when compared to the gold standard picture archiving and communication systems (PACSs).

Technique:We used BN (version 4.2) on an iPad mini to perform the same RPM steps, using the same terminology originally described by Baumgart. Conclusion: Bone Ninja is a simple validated deformity correction tool with accuracy comparable to PACS. It is a reasonable alternative to paper/ pencil cutouts for applying RPM for IM limb lengthening and deformity correction surgery.

Clinical significance: We proposed a digitised RPM for internal limb-lengthening surgeries, which is highly feasible and practical to use conveniently without the need for the traditional tedious paper-cutting procedure and related equipment.

Keywords: Bone lengthening, Intramedullary lengthening, Limb deformity, Motorised implantable nail, Reverse planning.

Strategies in Trauma and Limb Reconstruction (2019): 10.5005/jp-journals-10080-1425
\end{abstract}

\section{BACKGROUND}

Motorised fully implantable intramedullary (IM) lengthening devices had been gaining popularity all over the world. ${ }^{1-3}$ The advantages of better patient comfort and fewer complications in comparison to external fixators, in conjunction with improved devices, have led to this development. ${ }^{4}$

In contrast to external fixators, the lengthening axis of an IM device is determined by the diaphyseal anatomy and the nail entry point. Mechanical axis deviation may result if careful preoperative planning and surgical intervention are not completed. ${ }^{5}$

These nails have the advantage of better patient comfort, accurate control, and less complications commonly associated with external fixators, such as pin tract infection, and fracture risk after fixator removal. ${ }^{4,6}$

However, in comparison to external fixators in which the surgeon determines the lengthening axis by careful pin placement and frame construction, the lengthening axis for IM reconstruction is determined by the diaphyseal anatomy and the nail entry point. This may cause lateral mechanical axis deviation, proportional to the amount of lengthening, ${ }^{7}$ estimated to be $1 \mathrm{~mm}$ of shift (typically lateral) for every $1 \mathrm{~cm}$ lengthening. ${ }^{5}$ This is particularly relevant to femur lengthening due to divergent anatomic and mechanical axes, averaging $6-7^{\circ}$ divergence. ${ }^{8}$

To prevent this unplanned lateral shifting, Baumgart proposed the reverse planning method (RPM) ${ }^{9}$ that addresses the corrections needed to account for length, angulation, and translation created
1,6 Department of Orthopedics and Traumatology, Prince of Wales Hospital, Chinese University of Hong Kong, Hong Kong

${ }^{2}$ Department of Orthopedics, University of Utah, Salt Lake City, Utah, USA; Department of Orthopedic Surgery, International Center for Limb Lengthening, Rubin Institute for Advanced Orthopedics, Sinai Hospital of Baltimore, Baltimore, Maryland, USA

${ }^{3}$ Department of Orthopedic Surgery, Nemours/Alfred I. duPont Hospital for Children, Wilmington, Delaware, USA; Department of Orthopedic Surgery, International Center for Limb Lengthening, Rubin Institute for Advanced Orthopedics, Sinai Hospital of Baltimore, Baltimore, Maryland, USA

${ }^{4}$ Orthopedic Surgery Department, Al-Azhar University Hospitals, Cairo, Egypt

${ }^{5,7}$ Department of Orthopedic Surgery, International Center for Limb Lengthening, Rubin Institute for Advanced Orthopedics, Sinai Hospital of Baltimore, Baltimore, Maryland, USA

Corresponding Author: John E Herzenberg, Department of Orthopedic Surgery, International Center for Limb Lengthening, Rubin Institute for Advanced Orthopedics, Sinai Hospital of Baltimore, Baltimore, Maryland, USA, Phone: +1 410601 1724, e-mail: jherzenb@ lifebridgehealth.org

How to cite this article: Hung AL-H, McClure PK, Franzone JM, et al. Bone Ninja Mobile App for Reverse Planning Method in Internal Limb Deformity and Lengthening Surgery. Strategies Trauma Limb Reconstr 2019;14(2):72-76.

Source of support: Nil

() The Author(s). 2019 Open Access This article is distributed under the terms of the Creative Commons Attribution 4.0 International License (https://creativecommons. org/licenses/by-nc/4.0/), which permits unrestricted use, distribution, and non-commercial reproduction in any medium, provided you give appropriate credit to the original author(s) and the source, provide a link to the Creative Commons license, and indicate if changes were made. The Creative Commons Public Domain Dedication waiver (http://creativecommons.org/publicdomain/zero/1.0/) applies to the data made available in this article, unless otherwise stated. 
during lengthening along the nail axis. The RPM starts with the "ideal" final result of the projected lengthening and works backward to the preoperative status. The RPM plan serves as a reference to be recreated in the operating room.

The original RPM description by Baumgart calls for the use of life-size paper tracings of the bones, made off of $5^{\prime}$ long-standing films. In the era of digital X-ray, such long traditional films are usually no longer readily available. We propose a modified RPM using digital radiographs and a digital tool. Bone Ninja (BN), a mobile app, was developed for patient/physician education and is available for the iPad platform (Apple Inc., Cupertino, CA). This relatively inexpensive app allows for measurements and deformity correction planning without the need for scissors, paper, or expensive computer software packages, such as Trauma Cad (Brainlab, Westchester, IL). Bone Ninja has been shown to have similar accuracy for measurements of limb length and deformity angles when compared to the gold standard picture archiving and communication systems (PACSs)..$^{10}$ The aim of the current study was to report whether BN can also be feasible as a tool to teach RPM for implantable IM limb-lengthening device and for deformity correction surgery.

This study involved no human or animal subjects.

\section{TECHNIQUe}

We used BN (version 4.2) on an iPad mini to perform the same RPM steps, using the same terminology originally described by Baumgart. ${ }^{9}$ Extra substeps with detailed instructions and some modifications specific to the BN app were added to better illustrate how to reproduce all the RPM steps. Cases from our archives with single plane deformity in the femur and the tibia were chosen to better illustrate the process.

\section{Frontal Plane Varus Angular Deformity of the Femur- RPM for Retrograde Nailing (In the Presence of Normal Tibia)}

\section{Step 0 (Fig. 1A)}

Preoperative deformity measurements were performed on $\mathrm{BN}$ including the malalignment test, mechanical/anatomical joint inclination angles, the apex of deformity, the osteotomy level (OL),
Conflict of interest: $\mathrm{Dr}$ Herzenberg reports personal fees from OrthPediatrics, Orthofix, NuVasive Specialized Orthopedics, Smith and Nephew, and WishBone Medical, outside the submitted work. Dr Standard reports personal fees and other from NuVasive Specialized Orthopedics, Smith and Nephew, and Pega Medical, outside the submitted work. Dr Herzenberg, Dr McClure, and Dr Standard are employees of Sinai Hospital who publish the BN mobile app and their institution received support for an annual course for orthopedic surgeons from the following entities: Avitus Orthopaedics, CyMedica Orthopedics, DePuy Synthes, MHE Coalition, NuVasive Specialized Orthopedics, Orthofix, OrthoPediatrics, Paragon 28, Smith and Nephew, Stryker, Vilex, and Zimmer Biomet

and the magnitude of deformity and limb length discrepancy (LLD). A calibration tool (in this case a 1-inch steel ball, open slotted white arrow) is necessary to allow reproduction of the osteotomy site level and implant selection.

Step 1 (Fig. 1B)

Draw the mechanical axis of the tibia (vertical blue line, open black arrow) from the center of the ankle joint to the center of the knee joint and continue this line proximally beyond the projected final level of the femoral head. The new projected center of the hip joint lies at the intersection of this mechanical axis line and a horizontal line (horizontal blue line, closed black arrow) drawn above the existing femoral head by the desired added length.

\section{Step 2}

Identify the planned OL by measuring from the lateral condyle with the ruler and use the cut tool to outline the planned moving fragment (proximal fragment) (slotted open black arrow). Staying close to the cortical margins with the cut tool will generate a more natural image at the final step, permitting accurate measurements and reproduction.

\section{Step 3}

Draw the anatomic axis (green line) of the proximal segment. It should be long enough for easier selection as the moving part later (closed white arrow). Select the proper IM nail and align it exactly onto the anatomical axis (green line) of the proximal segment
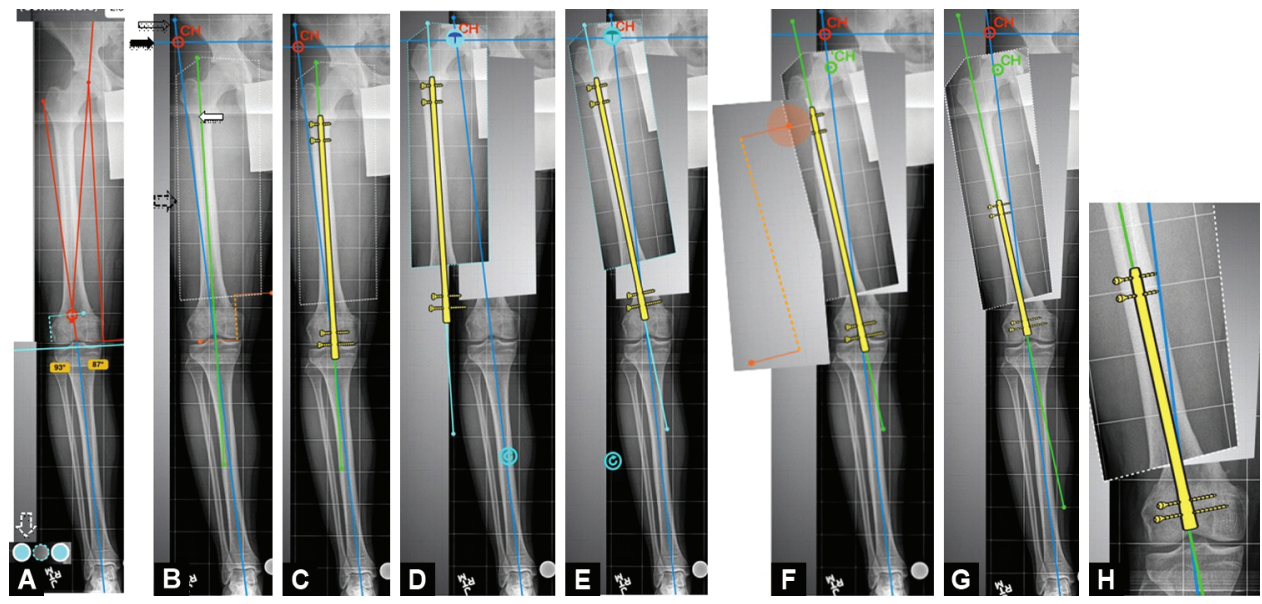

Figs 1 A to $\mathrm{H}$ : Reverse planning method for retrograde nailing of the femur. Steps A-H, see text for details (Copyright 2020, Rubin Institute for Advanced Orthopedics, Sinai Hospital of Baltimore): (A) Femoral deformity planning; (B) Open black arrow (vertical blue line); closed black arrow (horizontal blue line); slotted open black arrow (proximal osteotomised fragment); closed white arrow (green anatomical line); $\mathrm{CH}$, corrected hip center; (C) Implantable intramedullary nail fit to the green anatomical line; (D) Swivelling of implantable intramedullary nail at the newly corrected hip center (red CH); (E) Swivelled implantable intramedullary nail; (F) Repositioned implantable intramedullary nail with the final corrected hip center (green $\mathrm{CH}$ ); (G) Adjustment of size and length of the implantable intramedullary nail fitted to femoral anatomy; and (H) Final intraoperative alignment to be replicated 
(Fig. 1C). The line should project well beyond the margin of the moving fragment by approximately twice the planned lengthening in both directions to facilitate later steps. Multilevel deformity may be present in the bone; a plan to address or work around the deformity should be made accordingly (osteotomy vs shorter nail, etc.).

\section{Step 4}

Using the thumb tack tool, select the anatomic axis, the nail, and the proximal femur segment to group as one moving fragment and then move the three selected items as a group to align the femoral head into the new position (CH) (red center, Fig. 1D). Swivel the proximal segment of the femur using $\mathrm{CH}$ as the center of rotation by placing the thumb tack tool on it until the following three criteria are met (Fig. 1E):

- The nail projecting out of the proximal segment should enter the distal femoral segment at the $\mathrm{OL}$ and should exit through the apex of the femoral notch.

- The lateral proximal femoral angle should be within acceptable range.

- The nail follows a feasible surgical path. Excessive reaming of the cortical bone in the plan should prompt reevaluation of the $\mathrm{OL}$. A lower osteotomy will avoid this difficulty, but adequate size of the distal fragment must be maintained to allow locking screw and blocking screw fixation in the distal fragment.

\section{Step 5}

Deselect the anatomic axis line, move the grouped nail and the proximal bone segment distally along the nail axis (green line) until it reaches the distal fragment at the $\mathrm{OL}$, and then mark the new hip center (green $\mathrm{CH}$ ) (Fig. 1F). The size of the nail can be adjusted and measured. The recommended length of the nail is dependent on the presence of a sagittal bow in the femur and the desired amount of lengthening (Fig. 1G). Magnify the distal femur segment to measure its angulation and translation, which represent the final intraoperative goal to be replicated (Fig. 1H). When doing the actual case, it is imperative to insert blocking screws around the nail. ${ }^{11}$

The RPM using the BN app is not as feasible for antegrade nailing in frontal plane angular deformity of the femur, because there is very little room for changing the start point in the proximal femur. For example, moving the start point for a straight nail from the piriformis fossa to the greater trochanter will invariably create varus proximally.

\section{Frontal Plane Angular Deformity of the Tibia-RPM for Antegrade Nailing}

Note: Formal planning may not be needed if there is no tibial deformity, since the mechanical axis and the anatomic axis of the tibia are normally parallel to each other. If correction of a deformity is needed, the following method may be used.

\section{Step 0}

Step 0 is the same as the femoral planning method (Fig. 2A).

\section{Step 1 (Fig. 2B)}

Draw a vertical line (blue line, open black arrow) extending the femoral mechanical axis distally; this will define the planned mechanical axis of the leg at completion.

\section{Step 2}

The new ankle center (CA) is located along this line at a level distal to the native $C A$ by the amount of lengthening required (horizontal blue line, open white arrow). Choose an osteotomy line near the apex of the deformity, with consideration of adequate fixation of the proximal fragment (closed white arrow).

\section{Step 3}

Draw the anatomic axis (green line, open slotted white arrow). Choose internal nail-long (in the hardware tab), and adjust the
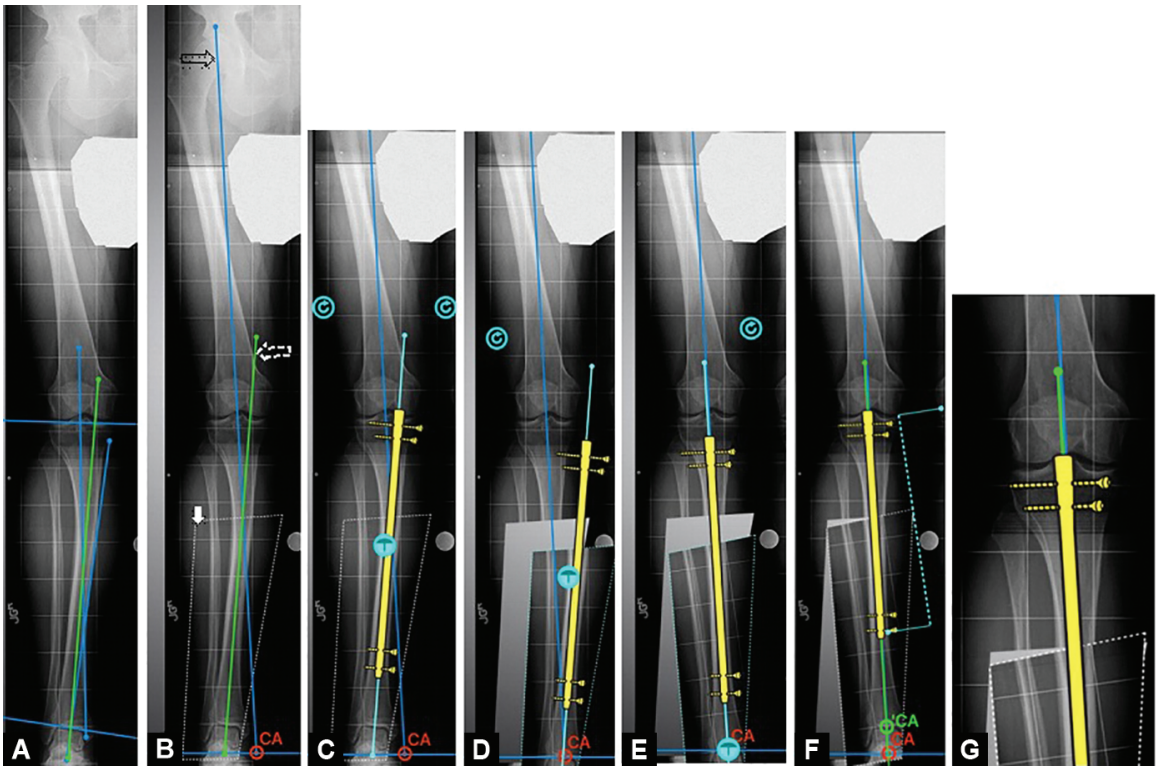

Figs 2A to G: Reverse planning method for ante-grade nailing of tibia. Steps A-K, see text for details (Copyright 2020, Rubin Institute for Advanced Orthopedics, Sinai Hospital of Baltimore): (A) Tibial deformity planning; (B) Open black arrow (vertical blue line); open white arrow (horizontal blue line); closed white arrow (distal tibial osteotomised fragment); slotted open white arrow (vertical green line); CA, corrected ankle center; (C) Implantable intramedullary nail fit to the green anatomical line; (D) Swiveling of the implantable intramedullary nail at the newly corrected ankle center (red CA); (E) Swiveled implantable intramedullary nail; (F) Repositioned implantable intramedullary nail with the final corrected ankle center (green CA); (G) Final intraoperative alignment to be replicated 


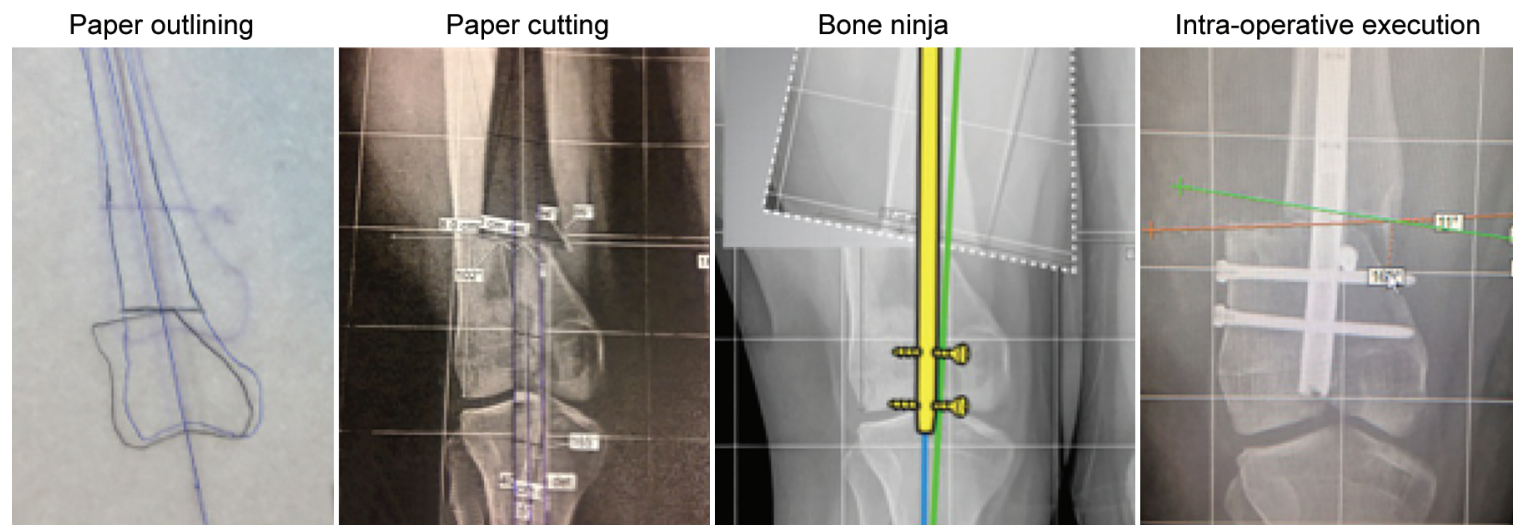

Fig. 3: Final alignment of the osteotomised fragment planned from different methods (Copyright 2020, Rubin Institute for Advanced Orthopedics, Sinai Hospital of Baltimore)

diameter of the nail, which will accommodate to the tibia canal, without excessive reaming after measuring the diameter of isthmus of the tibia and the nail. Align the nail exactly on the anatomical axis (green line) (Fig. 2C).

\section{Step 4}

Select the thumb tack tool, then the anatomical axis (green line), the nail, and the distal tibia segment. Place the center of the distal tibia to the point of CA as determined in step 2 (red dot) (Fig. 2D). Put the thumb tack tool at CA as the rotational axis, then swivel back such that the nail and its anatomical axis (green line) project onto the center of intercondylar of the tibia spine as the optimal entry point of nail insertion (Fig. 2E).

\section{Step 5}

Translate the nail together with the distal segment of the tibia along the anatomical axis by deselecting the anatomic axis, until contact of the fragments at the osteotomy site occurs. Mark the new (immediate postoperative) center of the ankle joint CA (green) (Fig. 2F). The size of nail can now be adjusted and measured. Magnify the osteotomy site to measure the translation and angulation angle (Fig. 2G). This is the image that should be reproduced in the operating room.

\section{Discussion}

Digital radiography has become the standard modality in most orthopedic centers over the past decade. ${ }^{12}$ The most commonly used system is the PACS that provides accurate, fast material transmission and saves hard copy storage space. ${ }^{13}$

In the field of deformity correction surgery, most PACSs do not allow for virtual bone-cutting and surgical simulation. ${ }^{13}$ Commercially available CAD/CAM programs like Trauma-Cad are designed for such purposes but are very expensive and not readily available in every centre. ${ }^{14}$ Therefore, many centers still rely on traditional methods of manual surgical simulation with paper and pencil.

The RPM was originally described based with the traditional method of paper, pencil, and scissors. ${ }^{9}$ However, the method can be cumbersome, and specialised equipment is still required for optimal execution of the RPM. Recently, Galal published a novel method of RPM that uses trigonometric formulas. While accurate, the introduction of trigonometry may be confusing to most orthopedic surgeons. ${ }^{15}$ In this paper, we demonstrate a high feasibility of BN to apply the RPM for IM limb-lengthening surgery, which is much easier to comprehend. In addition, BN is one of the few mobile applications that has been validated. ${ }^{10}$ The accuracy of deformity parameters after IM lengthening measured on the final manipulated fragments would appear to be comparable among all the planning methods, but validation would be desirable (Fig. 3). By using $\mathrm{BN}$, the original digital radiograph can be imported as an image file or by using the iPad camera. Planning can start on the original genuine digital images directly, obviating the need for tracing, cutting, and large-scale paper models.

An additional advantage to the digital method of reverse planning is the ability to modify the plan easily without additional resources (i.e., reprinting and retracing). At the completion of planning, it is not uncommon to need to optimise the OL to minimise cortical reaming. An osteotomy placed too high will generate the need for cortical reaming, while an osteotomy site too low may preclude adequate fixation in the distal fragment. The undo and redo functions of BN record all the previous steps, which is then easy to trace back and reedit without the need to replicate a new set of paper copies/drawing. Each planning step can also be exported easily as a high-quality jpeg image that enhances communication and discussion among surgeons and patients.

Bone Ninja is primarily a deformity planning program and has been equipped with sophisticated tools that allow accurate calibration, virtual osteotomy, and free manipulation of fragments in space, which are all important steps in RPM. The BN nudge tool facilitates very fine control of moving segments, allowing precise movements to specify point-to-point anatomy. Accuracy of measurements of distance or angulation is possible even while working in the small-screen iPad mini model, though we do prefer using the newer, larger-screen iPad models (ref. Whitaker).

Compared to other digital planning programs, BN is cheaper and more portable with easy access through the Apple App store. One disadvantage is that BN application is still limited to the iPad and is not available on other platforms such as Google Android.

\section{Limitations}

Due to a lack of additional virtual space distal to the level of the native radiograph, reverse planning with $\mathrm{BN}$ for the tibia requires modification of the initial image. Without adding additional space distally, multiple steps become difficult as the mobile fragment moves off of the available space. A simple modification is to capture 
an image using the camera function that includes additional space distal to the feet.

In short, BN is a simple, validated deformity correction tool with accuracy comparable to PACS. It is a reasonable alternative to paper/pencil cutouts for applying RPM for IM limb lengthening and deformity correction surgery.

\section{Conclusion}

Bone Ninja is a simple validated deformity correction tool with accuracy comparable to PACS. It is a reasonable alternative to paper/pencil cutouts for applying RPM for IM limb lengthening and deformity correction surgery.

\section{Clinical Significance}

We proposed a digitised RPM for internal limb lengthening surgeries, which is highly feasible and practical to use conveniently without the need for the traditional tedious paper-cutting procedure and related equipment.

\section{ACKnowledgments}

Miss Ka Yee Chan for figures modification and export. No financial support was received for this study.

\section{References}

1. Shabtai L, Specht SC, Standard SC, et al. Internal lengthening device for congenital femoral deficiency and fibular hemimelia. Clin Orthop Relat Res 2014;472(12):3860-3868. DOI: 10.1007/s11999-014-3572-3.

2. Dahl M, Birch J, Motorized Intramedullary Lengthenings 2006-2013 Using Baumgart's Technique. 23rd Annual Scientific Meeting of the Limb Lengthening and Reconstruction Society; New York, NY2013.

3. Baumgart R, Betz A, Schweiberer L. A fully implantable motorized intramedullary nail for limb lengthening and bone transport. Clin Orthop Relat Res 1997(343):135-143. DOI: 10.1097/00003086199710000-00023.

4. Black SR, Kwon MS, Cherkashin AM, et al. Lengthening in congenital femoral deficiency: a comparison of circular external fixation and a motorized intramedullary nail. J Bone Joint Surg Am 2015;97(17) 1432-1440. DOI: 10.2106/JBJS.N.00932.

5. Burghardt RD, Paley D, Specht SC, et al. The effect on mechanical axis deviation of femoral lengthening with an intramedullary telescopic nail. J Bone Joint Surg Br 2012;94(9):1241-1245. DOI: 10.1302/0301620X.94B9.28672.

6. Kocaoglu M, Eralp L, Bilen FE, et al. Fixator-assisted acute femoral deformity correction and consecutive lengthening over an intramedullary nail. J Bone Joint Surg Am 2009;91(1):152-159. DOI: 10.2106/JBJS.H.00114.

7. Burghardt RD, Herzenberg JE, Burghardt MH. Trigonometric analysis of the mechanical axis deviation induced by telescopic intramedullary femoral lengthening nails. J Appl Biomech 2011;27(4):385-391. DOI: 10.1123/jab.27.4.385.

8. Paley D, Herzenberg JE. Principles of deformity correction, vol. 1. Springer; 2002.

9. Baumgart R. The reverse planning method for lengthening of the lower limb using a straight intramedullary nail with or without deformity correction. A new method. Oper Orthop Traumatol 2009;21(2):221-233. DOI: 10.1007/s00064-009-1709-4.

10. Whitaker AT, Gesheff MG, Jauregui JJ, et al. Comparison of PACS and Bone Ninja mobile application for assessment of lower extremity limb length discrepancy and alignment. J Child Orthop 2016;10(5): 439-443. DOI: 10.1007/s11832-016-0761-5.

11. Muthusamy S, Rozbruch SR, Fragomen AT. The use of blocking screws with internal lengthening nail and reverse rule of thumb for blocking screws in limb lengthening and deformity correction surgery. Strategies Trauma Limb Reconstr 2016;11(3):199-205. DOI: 10.1007/s11751-016-0265-3.

12. Steinberg EL, Segev E, Drexler M, et al. Preoperative planning of orthopedic procedures using digitalized software systems. Isr Med Assoc J 2016;18(6):354-358.

13. Strickland NH. PACS (picture archiving and communication systems): filmless radiology. Arch Dis Child 2000;83(1):82-86. DOI: 10.1136/ adc.83.1.82.

14. Steinberg EL, Segev E. Pre-operative planning using the traumacad software system. US Radiol 2010;2(1):87-90.

15. Galal S. The resolution axis method (RAM) for lengthening of the femur with or without associated frontal plane deformity (a new method). Strategies Trauma Limb Reconstr 2018;13(2):109-118. DOI: 10.1007/s11751-018-0312-3. 